Results Healthy individuals andpatients with cystic fibrosis had robust antigen-specific memory $\mathrm{CD}^{+}{ }^{+} \mathrm{T}$ cell responses to Pseudomonas aeruginosa that not only contained a Th1 and Th17 component but also Th22 cells. In contrast to previous descriptions of human Th22 cells, these Pseudomonal-specific Th22 cells lacked the skin homing markers CCR4 or CCR10, although they did express the chemokine receptor CCR6 that would direct migration to damaged epithelial surfaces. Furthermore, IL22 production was evident in the lungs of CF patients colonised with PA. Healthy individuals and patients with cystic fibrosis had similar levels of Th22 and Th1 cells, but the patient group had significantly fewer Th17 cells in peripheral blood.

Conclusions MemoryTh22 cells specific to Pseudomonas aeruginosa are induced in both healthy individuals and patients with CF, with IL-22 secretion being demonstrated in the CF lung. These Th22 cells do not express tissue specificity for gut or skin sites and we thus hypothesise may have a role in respiratory defense. Along with Th17 cells, they may play an important role in the pathogenesis of pulmonary infection with this microbe in patients with cystic fibrosis.

\section{S101 PATHOGEN ASSOCIATED MOLECULAR PATTERNS IN CYSTIC FIBROSIS PATHOGENS: ANALYSIS OF PEPTIDOGLYCAN STRUCTURE}

${ }^{1} \mathrm{SC}$ Carnell, ${ }^{2} \mathrm{~J}$ Biboy, ${ }^{2} \mathrm{G}$ Cerardi, ${ }^{2} \mathrm{~B}$ Ville, ${ }^{2} \mathrm{C}$ Deleuse, ${ }^{2} \mathrm{R}$ Samain, ${ }^{2} \mathrm{D}$ Vollmer, ${ }^{2} \mathrm{CMA}$ Khan, ${ }^{2} \mathrm{~J}$ Gray, ${ }^{2} \mathrm{~W}$ Vollmer, ${ }^{1} \mathrm{~A}$ De Soyza; ${ }^{1}$ Institute for Cellular Medicine, University of Newcastle, Newcastle-upon-Tyne, UK; ${ }^{2}$ Centre for Bacterial Cell Biology, University of Newcastle, Newcastle-upon-Tyne, UK

\subsection{6/thoraxjnl-2013-204457.108}

Background Pathogen associated molecular patterns (PAMPS) are key virulence determinants in triggering immune mediated damage in cystic fibrosis. The lipopolysaccharide (LPS) in Pseudomonas aeruginosa strains isolated from CF-lungs has "CF specific" structural motifs not seen in other clinical isolates nor environmental strains. We have shown these motifs are also present in Burkholderia cepacia complex (BCC) species. We hypothesised that another key virulence determinant, peptidoglycan (PG), may show similar CF specific changes. PG is essential for bacterial cell wall stability and is the principle target for many antibiotics and for antimicrobial hydrolases of the innate immune system. Soluble PG fragments show biological activity interacting with NOD receptors.

Methods Burkholderia cepacia complex strains of interest were selected from the BCC international reference panel $(\mathrm{n}=8), P$. aeruginosa from a novel international reference panel and local repository $(\mathrm{n}=6)$ and Achromobacter xylosoxidans from a local repository $(n=6)$. After growth in LB media and SDS extraction the PG was digested and the resulting muropeptides were analysed by HPLC and MS. The profiles were compared to the well characterised E. coli PG.

Results $P$. aeruginosa PG was similar to the archetypical PG from E. coli. Strikingly, BCC spp. PG contained amidated muropeptides that have previously been described only in some Gram-positive bacteria. The extent of amidation varied between strains and did not map to either CF, other clinical or environmental origins. It was not linked to LPS chemotype nor BCC genomovars. A. xylosoxidans PG also contained novel muropeptide structures different to those seen in BCC.

Conclusions We describe for the first time novel and unexpected modifications in the PG of BCC and Achromobacter, which were present in all strains tested so far. These modifications may offer either a biological advantage against PG hydrolases or may be linked with modulating the host immune responses. Immunostimulatory data are presently being collected to investigate this aspect.

\section{S102 THE EFFECT OF RESPIRATORY VIRUSES ON THE LUNG MICROBIOME OF ADULTS WITH CYSTIC FIBROSIS}

${ }^{1}$ WG Flight, ${ }^{2}$ JR Marchesi, ${ }^{2} \mathrm{~A}$ Smith, ${ }^{2} \mathrm{P}$ Norville, ${ }^{3} \mathrm{KJ}$ Mutton, ${ }^{1} \mathrm{AK}$ Webb, ${ }^{1} \mathrm{RJ}$ Bright-Thomas, ${ }^{1} \mathrm{AM}$ Jones, ${ }^{2} \mathrm{E}$ Mahenthiralingam; 'University Hospital of South Manchester NHS Foundation Trust, Manchester, United Kingdom; ${ }^{2}$ School of Biosciences, Cardiff University, Cardiff, United Kingdom; ${ }^{3}$ Central Manchester University Hospitals NHS Foundation Trust, Manchester, United Kingdom

\subsection{6/thoraxjnl-2013-204457.109}

Introduction Viral respiratory infection (VRI) has been implicated in the pathogenesis of cystic fibrosis (CF) lung disease and it has been hypothesised that respiratory viruses may predispose CF patients to acquisition of bacterial pathogens. We performed a prospective observational study to determine whether VRI leads to a change in the respiratory microbiome of adults with CF.

Methods Participants provided sequential paired sputum samples over a ten month period. One sputum sample from each visit was processed using conventional culture. The second sample was analysed with $16 \mathrm{~S}$ rRNA gene pyrosequencing and ribosomal intergenic spacer analysis (RISA) to examine bacterial diversity. Polymerase chain reaction assays for nine respiratory viruses were also performed on the second sample. Study visits were classified as "stable," "viral" or "non-viral exacerbation." Generalised estimating equation models were used to examine differences between these groups of study visits. Statistical analysis accounted for repeated observations from individual patients.

Results Eighteen patients provided a total of 77 paired sputum samples over a median follow-up period of 290 days (range 62359). $23 / 46(50 \%)$ study visits were positive for a respiratory virus with rhinovirus accounting for 56\% of cases. 19/46 (41\%) study visits met the pre-specified criteria for pulmonary exacerbation of which 11 (58\%) were virus-positive and 8 (42\%) were virus-negative.

Conventional culture did not identify any new bacterial species during the study period. A mean of 5453 (SD 2847) reads per sample were detected by $16 \mathrm{~S}$ rRNA gene pyrosequencing. The mean Shannon Index was 0.59 (SD 0.58) with a mean richness of 15.1 (SD 7.9) genera. No consistent change in bacterial diversity indices or relative abundance of individual genera was seen in response to either VRI or non-viral exacerbations. The majority of patients had highly variable RISA and 16S rRNA gene profiles during follow-up. A subset of four patients (22\%) had a stable respiratory microbiome which was heavily dominated by Pseudomonas aeruginosa throughout despite episodes of VRI, pulmonary exacerbation and antibiotic therapy.

Conclusions Longitudinal change of the respiratory microbiome varies considerably among adults with CF. No consistent effect of either VRI or pulmonary exacerbation on the lung microbiome was observed.

\section{S103 NEUTROPHIL TRANS-EPITHELIAL MIGRATION IN CYSTIC FIBROSIS AIRWAYS}

${ }^{1} \mathrm{C}$ Rebeyrol, ${ }^{2} \mathrm{JC}$ Porter; ${ }^{1}$ Centre for Inflammation and Tissue Repair, University College London, London, United Kingdom; ${ }^{2}$ Respiratory Medicine Service, University College London Hospitals NHS Trust, London, United Kingdom

10.1136/thoraxjnl-2013-204457.110 